\title{
Approximate-Karush-Kuhn-Tucker Conditions and Interval Valued Vector Variational Inequalities
}

\author{
KIN KEUNG LAI \\ College of Economics \\ Shenzhen University \\ Shenzhen, 518060 \\ CHINA
}

\author{
SANJEEV KUMAR SINGH \\ Department of Mathematics \\ Institute of Science \\ Banaras Hindu University \\ Varanasi, 221005 \\ INDIA
}

\author{
SHASHI KANT MISHRA \\ Department of Mathematics \\ Institute of Science \\ Banaras Hindu University \\ Varanasi, 221005 \\ INDIA
}

\begin{abstract}
This Article deals with the Approximate Karush-Kuhn-Tucker (AKKT) optimality conditions for interval valued multiobjective function as a generalization of Karush-Kuhn-Tucker optimality conditions. Further, we establish relationship between vector variational inequality problems and multiobjective interval valued optimization problems under the assumption of LU convex smooth and non-smooth objective functions.
\end{abstract}

Key-Words: Interval valued functions, Optimality conditions, Vector variational inequality.

Received: October 27, 2019. Revised: May 12, 2020. Accepted: May 24, 2020. Published: June 4, 2020.

\section{Introduction}

The sequential optimality conditions, for example, Approximate-Karush-Kuhn-Tucker $(A K K T)$ condition [17] needs the existence of a sequence $\left\{x^{k}\right\}$, which is converging to some $x^{*}$ with the condition that $x^{k}$ is a Karush-Kuhn-Tucker $(K K T)$ point for every natural number $k$, also there should be an appropriate sequence of Lagrange multipliers with the property that gradient of the Lagrangian function at $x^{k}$ converges to zero.

The $K K T$ conditions [12] play a vital role to solve nonlinear optimization problems, both for scalar optimization and for multiobjective optimization problems. Numerically, the optimality conditions based on the sequence of iterands, which is known as sequential optimality conditions, do not need any constraint qualification [14].

Lions and Stampacchia [13] introduced the concept of variational inequality problem. Further, Giannessi [7] introduced the concept of vector variational inequalities. Variational inequalities and vector variational inequalities play an important role in deriving necessary and sufficient optimality conditions for scalar and vector optimization problems. Mastroeni [11] established relationship between $K K T$ conditions and variational and vector variational inequalities. Further, Haeser and Schuverdt [10] established the relationship between sequential optimality conditions and variational inequalities. Haeser and Schuverdt [10] introduced necessary $A K K T$ condition for a solution to the continuous variational inequality problem. Recently, Giorgi et al. [9] generalized the sequential optimality conditions from scalar optimization problem to multiobjective optimization problem. Further, Giorgi et al. [9] showed that these $A K K T$ conditions are necessary for a point to be a weak efficient solution and sufficient under some convexity and affinity assumptions.

The real world optimization problems associate some uncertain data due to measurement errors. Two optimization models came into existence to handle with the uncertain data. First is robust optimization and second one is interval valued optimization [6]. Interval analysis [5] as well as interval valued Optimization are the growing branch of mathematics in this computer age with the effect of rounding errors due to uncertain data. Wu [2] established relationship between $K K T$ optimality conditions and interval valued objective functions. Wu [4] derived Wolfe duality results for interval valued optimization problems. Further, Wu [3] studied KKT optimality conditions in multiobjective programming problems with interval valued objective function.

Recently, Laha and Mishra [18] established some results in vector optimization problems and vector variational inequalities involving locally Lipschitz functions.

In this paper, we introduce Approximate $K K T$ optimality conditions for multiobjective interval valued objective function as a generalization of $K K T$ optimality conditions. The multiobjective function is associated with the vector variational inequality prob- 
lem. In addition to that, we establish relationship between vector variational inequality problems and multiobjective interval valued Optimization problems under the assumption of $L U$-convex smooth and nonsmooth objective functions.

Motivated by the work of $\mathrm{Wu}$ [3], Andreani et al. [16], Haeser and Schuverdt [10], Mastroeni [11] and Giorgi et al. [9], we introduce Approximate-KarushKuhn-Tucker optimality conditions for interval valued objective function and discuss the sufficiency of $A K K T$ conditions for the interval valued problems and generalize its definition to the structure of vector variational inequality problems.

The organization of this paper is as follows: In Section 2, we collected some basic definitions and results. In Section 3, we develop sequential optimality conditions as $A K K T$ conditions for interval valued vector variational inequality problem and proved sufficiency with $L U$-convex and affine conditions.

\section{Preliminaries}

\subsection{Interval Analysis}

We collect some basic concepts and essential definitions related to interval valued functions.

We denote by $\mathcal{I}$ the class of all closed intervals in $\mathbb{R}$. Let $U=\left[u^{L}, u^{U}\right]$, where $u^{L}$ and $u^{U}$ denotes the lower and upper bounds of $U$, respectively. Let $U=\left[u^{L}, u^{U}\right]$ and $V=\left[v^{L}, v^{U}\right]$ be in $\mathcal{I}$, then, we have

(i) $U+V=\{u+v: u \in U, v \in V\}=\left[u^{L}+\right.$ $\left.v^{L}, u^{U}+v^{U}\right]$,

(ii) $-U=\{-u: u \in U\}=\left[-u^{U},-u^{L}\right]$,

(iii) $U-V=U+(-V)=\left[u^{L}-v^{U}, u^{U}-v^{L}\right]$,

(iv) $t U=\{t u: u \in U\}=$ $\left\{\begin{array}{l}{\left[t u^{L}, t u^{U}\right] \quad \text { if } \quad t \geq 0} \\ {\left[t u^{U}, t u^{L}\right] \quad \text { for } \quad t<0}\end{array}\right.$ where $t$ is a real number.

we refer to Moore [5], for further details on interval analysis .

Suppose that $U \subseteq \mathbb{R}^{n}$ and $V \subseteq \mathbb{R}^{n}$, then the Hausdorff metric between $U$ and $V$ is denoted and defined by

$d_{H}(U, V)=\max \left\{\sup _{u \in U} \inf _{v \in V}\|u-v\|, \sup _{v \in V} \inf _{u \in U}\|u-v\|\right\}$,

where $\|$.$\| is an Euclidean norm.$

Let $U=\left[u^{L}, u^{U}\right]$ and $B=\left[v^{L}, v^{U}\right]$ be two closed intervals, then it is easy to prove that

$$
d_{H}(U, V)=\max \left\{\left|u^{L}-v^{L}\right|,\left|u^{U}-v^{U}\right|\right\} .
$$

Let $\left\{U_{n}=\left[u_{n}^{L}, u_{n}^{U}\right]\right\}$ and $U$ be closed intervals in $\mathbb{R}$, then the sequence of closed interval $\left\{U_{n}\right\}$ converges to $U$, if for every $\epsilon>0$, there exists $N>0$ such that, for $n>N$, we have $d_{H}\left(U_{n}, U\right)<\epsilon$. Wu [2] proved that

$$
\begin{gathered}
\lim _{n \rightarrow \infty} U_{n}=U \text { if and only if } \lim _{n \rightarrow \infty} u_{n}^{L}=u^{L} \text { and } \\
\lim _{n \rightarrow \infty} u_{n}^{U}=u^{U} .
\end{gathered}
$$

The function $\phi: \mathbb{R}^{n} \rightarrow \mathcal{I}$ is called interval valued function, this means $\phi(x)=\phi\left(x_{1}, \cdots, x_{n}\right)$ is a closed interval in $\mathbb{R}$ for each $x \in \mathbb{R}^{n}$. $\phi$ can be written as $\phi(x)=\left[\phi^{L}(x), \phi^{U}(x)\right]$, where $\phi^{L}$ and $\phi^{U}$ are two real valued functions defined on $\mathbb{R}^{n}$ such that $\phi^{L}(x) \leq \phi^{U}(x), \forall x \in \mathbb{R}^{n}$.

$\mathrm{Wu}$ [2] discussed limit and continuity of interval valued functions. Let $\phi$ be an interval valued function defined on $\mathbb{R}^{n}$ and $U=\left[u^{L}, u^{U}\right]$ be an interval in $\mathbb{R}$, we say

$$
\begin{gathered}
\lim _{x \rightarrow a} \phi(x)=U, \text { if and only if } \lim _{x \rightarrow a} \phi^{L}(x)=u^{L} \text { and } \\
\lim _{x \rightarrow a} \phi^{U}(x)=u^{U} .
\end{gathered}
$$

The interval valued function $f$ defined on $\mathbb{R}^{n}$ is said to be continuous at $a \in \mathbb{R}^{n}$ if

$$
\lim _{x \rightarrow a} \phi(x)=\phi(a) .
$$

Proposition 2.1 [3] Suppose $\phi$ is an interval valued function defined on $\mathbb{R}^{n}$, then $\phi$ is continuous at a $\in$ $\mathbb{R}^{n}$ if and only if $\phi^{L}$ and $\phi^{U}$ are continuous at a.

Definition 2.1 [3] Suppose $K$ is an open set in $\mathbb{R}$ The interval valued function $\phi: K \rightarrow \mathcal{I}$ with $\phi(x)=$ $\left[\phi^{L}(x), \phi^{U}(x)\right]$ is called weakly differentiable at $x^{0}$ if the real valued functions $\phi^{L}$ and $\phi^{U}$ are differentiable at $x^{0}$ (in the ordinary sense).

For $U, V \in \mathcal{I}$, if there exists a $W \in \mathcal{I}$ such that $U=$ $V+W$, then $W$ is called the Hukuhara difference of $U$ and $V$. Also, $W$ can be written as $W=U \ominus V$, considering the Hukuhara difference $W$ exists, which means that $u^{L}-v^{L} \leq u^{U}-v^{U}$ and $W=\left[u^{L}-\right.$ $\left.v^{L}, u^{U}-v^{U}\right]$.

Proposition 2.2 [3] Suppose $U=\left[u^{L}, u^{U}\right]$ and $V=$ $\left[v^{L}, v^{U}\right]$ are two closed intervals in $\mathbb{R}$. If $u^{L}-v^{L} \leq$ $u^{U}-v^{U}$, then the Hukuhara difference $W$ exists and $W=\left[u^{L}-v^{L}, u^{U}-v^{U}\right]$.

Definition 2.2 [3] Suppose $K$ is an open set in $\mathbb{R}$. The interval valued function $\phi: K \rightarrow \mathcal{I}$ is called 
$H$-differentiable at $x^{0}$ if there exists a closed interval $U\left(x^{0}\right) \in \mathcal{I}$ such that the limits

$$
\begin{gathered}
\lim _{h \rightarrow 0_{+}} \frac{\phi\left(x^{0}+h\right) \ominus \phi\left(x^{0}\right)}{h} \text { and } \\
\lim _{h \rightarrow 0_{+}} \frac{\phi\left(x^{0}\right) \ominus \phi\left(x^{0}-h\right)}{h}
\end{gathered}
$$

both exist and equal to $U\left(x^{0}\right)$, which is called the $H-$ derivative of $\phi$ at $x^{0}$.

\subsection{Solution Concepts}

Suppose $U=\left[u^{L}, u^{U}\right]$ and $V=\left[v^{L}, v^{U}\right]$ are two closed intervals in $\mathbb{R}$. We write $U \preceq{ }_{L U} V$ if and only if $u^{L} \leq v^{L}$ and $u^{U} \leq v^{U}$.

Consider multiobjective programming problem with multiple interval valued objective functions

$$
(M I V P) \quad \min \phi(x)=\left(\phi_{1}(x), \cdots, \phi_{p}(x)\right)
$$

subject to $x=\left(x_{1}, \cdots, x_{n}\right) \in K \subseteq \mathbb{R}^{n}$,

where each

$\phi_{k}(x)=\left[\phi_{k}^{L}(x), \phi_{k}^{U}(x)\right]$ is an interval valued function for $k=1, \cdots, p$.

We write $U \prec_{L U} V$ if and only if $U \preceq_{L U} V$ and $U \neq V$. We say $U=\left(U_{1}, \cdots, U_{p}\right)$ is an interval valued vector if each component $U_{k}=\left[u_{k}^{L}, u_{k}^{U}\right]$ is closed interval for $k=1, \cdots, p$. Suppose $U=$ $\left(U_{1}, \cdots, U_{p}\right)$ and $V=\left(V_{1}, \cdots, V_{p}\right)$ be two interval valued vectors. We write $U \preceq_{L U} V$ if and only if $U_{k} \preceq_{L U} V_{k} \forall k=1, \cdots, p$, and $U \prec_{L U} V$ if and only if $U_{k} \preceq_{L U} V_{k}, \forall k=1, \cdots, p$ and $U_{q} \prec_{L U} V_{q}$ for at least one $q$. Suppose $x^{*}$ is a feasible solution of $(M I V P)$, then $\phi\left(x^{*}\right)$ is an interval valued vector. The concepts of Pareto optimal (efficient) solution is given below.

Definition 2.3 [2] Suppose $x^{0}$ is a feasible solution to the problem $(M I V P)$.

(i) $x^{0}$ is said to be an efficient solution to the problem $(M I V P)$ if there exists no $\bar{x}$ such that $\phi(\bar{x}) \prec_{L U} \phi\left(x^{0}\right)$.

(ii) $x^{0}$ is said to be a strong efficient solution to the problem $(M I V P)$ if there exists no $\bar{x}$ such that $\phi(\bar{x}) \preceq_{L U} \phi\left(x^{0}\right)$.

(iii) $x^{0}$ is said to be a weak efficient solution to the problem $(M I V P)$ if there exists no $\bar{x}$ such that $\phi_{k}(\bar{x}) \prec_{L U} \phi_{k}\left(x^{0}\right) \forall k=1, \cdots, p$.

Definition 2.4 [2] Suppose $x^{0}$ is feasible solution of the problem $(M I V P) . x^{0}$ is said to be local weak efficient solution of the problem $(M I V P)$, if there exists a neighborhood $N$ of $x^{0}$ such that for all $\bar{x} \in K \cap N$, then the following cannot satisfy for any $k=1, \cdots, p$

$$
\phi_{k}(\bar{x}) \prec_{L U} \phi_{k}\left(x^{0}\right) .
$$

Zhang et al. [19] defined the concepts of local quasi efficient and local weak quasi efficient solutions for the problem $(M I V P)$.

Definition 2.5 Suppose $x^{0}$ is feasible solution of the problem $(M I V P) . x^{0}$ is said to be local quasi efficient solution of the problem $(M I V P)$, if there exist $\beta \in \operatorname{int}\left(\mathbb{R}_{+}^{p}\right)$ and a neighborhood $N$ of $x^{0}$ such that for all $\bar{x} \in K \cap N$, then the following cannot satisfy

$$
\phi(\bar{x})+\beta\left\|\bar{x}-x^{0}\right\| \prec_{L U} \phi\left(x^{0}\right) .
$$

Definition 2.6 Suppose $x^{0}$ is feasible solution of the problem $(M I V P) \cdot x^{0}$ is said to be local weak quasi efficient solution of the problem $(M I V P)$, if there exist $\beta_{k} \in \operatorname{int}\left(\mathbb{R}_{+}^{p}\right)$ and a neighborhood $N$ of $x^{0}$ such that for all $\bar{x} \in K \cap N$, then the following cannot satisfy $k=1, \cdots, p$

$$
\phi_{k}(\bar{x})+\beta_{k}\left\|\bar{x}-x^{0}\right\| \prec_{L U} \phi_{k}\left(x^{0}\right) .
$$

\subsection{Optimization Problems}

We recall some basic and essential definitions. The open (closed) ball with center at $y^{0} \in \mathbb{R}^{n}$ with radius $\delta>0$ is denoted by $B\left(y^{0}, \delta\right)\left(\bar{B}\left(y^{0}, \delta\right)\right)$. We denote $\mathbb{R}_{+}^{n}$ as the non-negative orthant of $\mathbb{R}^{n}$. We also denote $c_{+}=\max \{0, c\}, c_{+}^{2}=\left(c_{+}\right)^{2}$, where $c \in \mathbb{R}$. The notation $\|\cdot\|$ is the Euclidean norm of $\mathbb{R}^{n}$. For $y, z \in$ $\mathbb{R}^{n}, y \leq z$ iff $y_{i} \leq z_{i}$, for $i=1, \ldots, n ; y<z, y_{i}<z_{i}$, for $i=1, \ldots, n$.

Let $K$ be real Banach Space with a norm $\|$.$\| and$ $K^{*}$ be its dual space with a norm $\|\cdot\|^{*}$. Let $X$ be a non-empty open convex subset of $K, \mathcal{F}: K \rightarrow 2^{K^{*}}$ be a set-valued mapping from real Banach space to the family of non-empty subsets of $K^{*}$. The following definitions and results are extracted from $[8,1]$ to resolve difficulties during the derivation of upcoming results.

Definition 2.7 (Generalized directional derivative) Suppose $\phi$ is a locally Lipschitz function at a given point $a \in K$ and $b$ be any other vector in $K$. Generalized directional derivative of $\phi$ at $a$ in the direction of $b$, denoted by $\phi^{0}(a ; b)$, is defined by

$$
\phi^{0}(a ; b)=\limsup _{y \rightarrow a, t \downarrow 0} \frac{\phi(y+t b)-\phi(y)}{t} .
$$

Definition 2.8 (Clarke's generalized subdifferential) Suppose $\phi$ is a locally Lipschitz function at a given 
point $a \in K$ and $b$ be any other vector in $K$. The Clarke's generalized subdifferential of $\phi$ at a, denoted by $\partial^{c} \phi(a)$, is defined by

$$
\partial^{c} \phi(a)=\left\{\xi \in K^{*}: \phi^{0}(a ; b) \geq\langle\xi, b\rangle, \forall b \in K\right\} .
$$

Next, we gather some properties related to Clarke's generalized subdifferential which can be found in [8].

Proposition 2.3 Let $\phi: \mathbb{R}^{n} \rightarrow \mathbb{R}$ be locally Lipschitz at $x$ with constant $L$. Then

1. $\partial^{c} \phi(x)$ is a nonempty, convex and compact set such that $\partial^{c} \phi(x) \subset B(0 ; L)$,

2. $\phi^{0}(x, v)=\max \left\{\langle v, \xi\rangle \mid \xi \in \partial^{c} \phi(x)\right\} \forall v \in \mathbb{R}^{n}$,

3. the map $\partial^{c} \phi(\cdot): \mathbb{R}^{n} \rightarrow \mathcal{P}\left(\mathbb{R}^{n}\right)$ is upper semicontinuous, where $\mathcal{P}\left(\mathbb{R}^{n}\right)$ denotes the power set of $\mathbb{R}^{n}$,

4. if $\phi$ is differentiable at $x$, then $\nabla \phi(x) \in \partial^{c} \phi(x)$,

5. if $\phi$ attains its extremum at $x$, then $0 \in \partial^{c} \phi(x)$.

Proposition 2.4 Let the functions $\phi_{i}: \mathbb{R}^{n} \rightarrow \mathbb{R}$ be locally Lipschitz at $x$ for $i=1,2, \ldots, k$, then for $\lambda_{i} \in$ $\mathbb{R}$

$$
\partial^{c}\left(\sum_{i=1}^{k} \lambda_{i} \phi_{i}\right)(x) \subset \sum_{i=1}^{k} \lambda_{i} \partial^{c} \phi_{i}(x) .
$$

Proposition 2.5 If $\phi_{1}$ and $\phi_{2}$ are locally Lipschitz at $x \in \mathbb{R}^{n}$, then the function $\phi_{1} \phi_{2}$ is locally Lipschitz at $x$ and

$$
\partial^{c}\left(\phi_{1} \phi_{2}\right)(x) \subset \partial^{c} \phi_{1}(x) \phi_{2}(x)+\phi_{1}(x) \partial^{c} \phi_{2}(x) .
$$

\subsection{Approximate-Karush-Kuhn-Tucker con- ditions $(A K K T)$ [17]}

We consider the nonlinear constrained optimization problems $(O P)$.

(OP) Minimize $\phi(x)$ subject to

$$
x \in P=\left\{x \in \mathbb{R}^{n}: g(x) \leq 0, h(x)=0\right\},
$$

where $\phi: \mathbb{R}^{n} \rightarrow \mathbb{R}, g: \mathbb{R}^{n} \rightarrow \mathbb{R}^{m}, h: \mathbb{R}^{n} \rightarrow \mathbb{R}^{r}$ are smooth functions. We say a feasible point $x^{0}$ satisfies $(A K K T)$ conditions, if there exists a sequences $\left(\mu^{k}, \tau^{k}\right) \subset \mathbb{R}_{+}^{m} \times \mathbb{R}^{r},\left\{x^{k}\right\} \subset \mathbb{R}^{n}$ converging to $x^{0}$ and satisfies the following:

$$
\begin{gathered}
\lim _{k \rightarrow \infty} \| \nabla \phi\left(x^{k}\right)+\sum_{j=1}^{m} \mu_{j}^{k} \nabla g_{j}\left(x^{k}\right) \\
+\sum_{l=1}^{r} \tau_{l}^{k} \nabla h_{l}\left(x^{k}\right) \|=0, \\
g_{j}\left(x^{*}\right)<0 \Longrightarrow \mu_{j}^{k}=0 \text { for sufficiently large } k, \\
j=1, \ldots, m .
\end{gathered}
$$

Let $P$ be non-empty and convex subset of $\mathbb{R}^{n}$ and $F: \mathbb{R}^{n} \rightarrow \mathbb{R}^{n}$ be a continuous map, then variational inequality (VI) problem [13] is stated as follows:

$$
\begin{aligned}
& V I(F, P) \quad \text { find } y^{0} \in P, \\
& \text { such that }\left\langle F\left(y^{0}\right), y-y^{0}\right\rangle \geq 0, \forall y \in P .
\end{aligned}
$$

Wu [2] established the Karush-Kuhn-Tucker (KKT) optimality conditions for interval valued optimization problem.

$$
(I V O P) \quad \min \phi(x)=\left[\phi^{L}(x), \phi^{U}(x)\right]
$$

$$
\text { subject to } g_{i}(x) \leq 0, i=1,2, \cdots, m \text {. }
$$

Let $P=\left\{x \in \mathbb{R}^{n}: g_{i}(x) \leq 0, i=1,2, \cdots, m\right\}$ be a feasible region of problem $(I V O P)$ and a point $\bar{x} \in P$. We say that the real valued function $g_{i}, i=$ $1,2, \cdots, m$ satisfy the $K K T$ conditions at $x^{0}$ if $g_{i}$ are convex on $\mathbb{R}^{n}$ and continuously differentiable at $x^{0} \forall i=1,2, \cdots, m$. The $K K T$ optimality conditions for problem $(I V O P)$ is given as follows.

Theorem 2.1 Let $g_{i}, i=1,2, \cdots, m$ be the real valued constraint functions which satisfy the KKT conditions at $x^{0}$ and the interval valued objective function $\phi: \mathbb{R}^{n} \rightarrow \mathcal{I}$ is $L U$-convex and weakly continuously differentiable at $x^{0}$, if there exist Lagrange multipliers $0<\lambda^{L}, \lambda^{U} \in \mathbb{R}$ and $0 \leq \mu_{j} \in \mathbb{R}, j=1,2, \cdots, m$, such that

(i) $\lambda^{L} \nabla \phi^{L}\left(x^{0}\right)+\lambda^{U} \nabla \phi^{U}\left(x^{0}\right)+\sum_{j=1}^{m} \mu_{j} \nabla g_{j}\left(x^{0}\right)=$ 0 ;

(ii) $\mu_{j} g_{j}\left(x^{0}\right)=0, \forall j=1,2, \cdots, m$,

then $x^{0}$ is a Pareto optimal solution of problem $(I V O P)$.

The vector variational inequality problem for interval valued function is given in Zhang et al. [19]:

$(V V I P)$ Find a point $x^{0} \in P$ such that there exist no $x \in P$ such that

$$
\begin{aligned}
& \left(\left\langle\nabla \phi_{1}^{L}\left(x^{0}\right)+\nabla \phi_{1}^{U}\left(x^{0}\right), x-x^{0}\right\rangle, \cdots,\right. \\
& \left.\left\langle\nabla \phi_{p}^{L}\left(x^{0}\right)+\nabla \phi_{p}^{U}\left(x^{0}\right), x-x^{0}\right\rangle\right)^{T} \leq 0 .
\end{aligned}
$$

\section{Approximate $K K T$ conditions and Vector Variational Inequalities}

We consider following vector optimization problem with interval valued objective functions.

(VVI-IVOP) Minimize $\left\langle F\left(x^{0}\right), x\right\rangle$, where $\left\langle F\left(x^{0}\right), x\right\rangle=\left(\left\langle F_{1}\left(x^{0}\right), x\right\rangle, \ldots,\left\langle F_{p}\left(x^{0}\right), x\right\rangle\right)$, subject to $x \in P$, 
where each $F_{k}\left(x^{0}\right)=\left[F_{k}^{L}\left(x^{0}\right), F_{k}^{U}\left(x^{0}\right)\right]$ is an interval valued function for $k=1,2, \cdots, p$ and feasible set $P$ is subset of $\mathbb{R}^{n}$.

A point $x^{0} \in P$ is an efficient solution of $V V I-$ $I V O P$ if and only if there exists no $x \in P$ such that $F(x) \leq F\left(x^{0}\right), F(x) \neq F\left(x^{0}\right)$. The set of all efficient solution of $V V I-I V O P$ is denoted by $\operatorname{Min}(F, P)$.

We establish the Approximate-Karush-KuhnTucker necessary and sufficient optimality conditions for vector variational inequality problems.

Definition 3.1 (AKKT-VVI-IVOP Conditions) The Approximate-Karush-Kuhn-Tucker conditions are satisfied for $V V I-I V O P$ at a feasible point $x^{0} \in P$ if and only if there exist sequences $\left(x^{k}\right) \subset \mathbb{R}^{n}$ and $\left(\lambda^{L, k}, \lambda^{U, k} \mu^{k}, \tau^{k}\right) \subset \mathbb{R}_{+}^{p} \times \mathbb{R}_{+}^{p} \times \mathbb{R}_{+}^{m} \times \mathbb{R}^{r}$, such that

(Al) $x^{k} \rightarrow x^{0}$,

$$
\begin{aligned}
& \sum_{i=1}^{p} \lambda_{i}^{L, k} F_{i}^{L}\left(x^{k}\right)+\sum_{i=1}^{p} \lambda_{i}^{L, k} F_{i}^{U}\left(x^{k}\right)+ \\
& \sum_{j=1}^{m} \mu_{j}^{k} \nabla g_{j}\left(x^{k}\right)+\sum_{l=1}^{r} \tau_{l}^{k} \nabla h_{l}\left(x^{k}\right) \rightarrow 0 \\
& \sum_{i=1}^{p} \lambda_{i}^{L, k}+\sum_{i=1}^{p} \lambda_{i}^{U, k}=1,
\end{aligned}
$$

(A3) $g_{j}\left(x^{0}\right)<0 \Longrightarrow \mu_{j}^{k}=0$, for sufficiently large $k, j=1,2, \cdots, m$.

The points satisfying $A K K T-V V I-I V O P$ conditions are called $A K K T-V V I$ points. Note that the sequence $x^{k}$ is not necessarily in feasible set. we scalarize the following nonsmooth function to establish necessary optimality conditions for the problem $V V I-I V O P,:$

$$
\mathfrak{F}: \mathbb{R}^{p} \rightarrow \mathbb{R} \text {, defined by } \mathfrak{F}(y)=\max \left\{y_{i}\right\},
$$

clearly $\mathfrak{F}(y) \leq 0 \Leftrightarrow y \leq 0$ and $\mathfrak{F}(y)<0 \Leftrightarrow y<0$.

We presented a lemma motivated by result from Giorgi et al. [9]:

Lemma 3.1 If $x^{0}$ is solution of interval valued $\operatorname{VVI}(F, P)$, then $x^{0}$ is solution of $\operatorname{Min}(\mathfrak{F}(F()-$. $\left.\left.F\left(x^{0}\right)\right), P\right)$.

Proof: Suppose $x^{0}$ is solution of $V V I-I V O P$, then there exist no $x$ such that

$$
\begin{aligned}
\left(\left\langleF_{1}^{L}\left(x^{0}\right)+\right.\right. & \left.F_{1}^{U}\left(x^{0}\right), x\right\rangle, \cdots \\
& \left.\left\langle F_{p}^{L}\left(x^{0}\right)+F_{p}^{U}\left(x^{0}\right), x\right\rangle\right)^{T} \leq 0 .
\end{aligned}
$$

Suppose on contrary $x^{0} \notin \operatorname{Min}\left(\mathfrak{F}\left(F()-.F\left(x^{0}\right)\right), P\right)$, then there exists $\bar{a}$ such that $\mathfrak{F}\left(F_{k}(a)-F_{k}\left(x^{0}\right) \prec_{L U}\right.$
$\mathfrak{F}\left(F_{k}\left(x^{0}\right)-F_{k}\left(x^{0}\right)=0, \forall k=1, \cdots, p\right.$. This follows that

$$
F_{k}(a) \prec_{L U} F_{k}\left(x^{0}\right) \forall k=1, \cdots, p,
$$

which is contradiction to the supposition. This completes the proof.

The following necessary optimality conditions for multiobjective optimization problem for local efficient solution of $(M O P)$ to be a Approximate-KarushKuhn-Tucker point will be helpful to develop the proof.

Theorem 3.1 If $x^{0} \in P$ is solution to the $V V I(F, P)$, then $x^{0}$ satisfies the AKKT - VVIIVOP conditions.

Proof: Since $x^{0}$ is local solution of $V V I(F, P)$, so by Lemma 3.1, there exists $\delta>0$, such that $x^{0} \in$ $\operatorname{Min}\left\{\mathfrak{F}\left(F(\cdot)-F\left(x^{0}\right)\right), P \cap \bar{B}\left(x^{0}, \delta\right)\right\}, F(x)=$ $\left[F^{L}(x), F^{U}(x)\right]$.

Suppose $x^{0}$ is unique solution of the problem

$$
\begin{gathered}
\text { Min } \mathfrak{F}\left(F(x)-F\left(x^{0}\right)\right)+\frac{1}{2}\left\|x-x^{0}\right\|^{2}, \\
\text { subject to } x \in P \cap \bar{B}\left(x^{0}, \delta\right), \\
\text { where } F(x)=\left[F^{L}(x), F^{U}(x)\right] .
\end{gathered}
$$

We define the following function:

$$
\begin{gathered}
\varphi_{k}(x)=\mathfrak{F}\left(F(x)-F\left(x^{0}\right)\right)+\frac{1}{2}\left\|x-x^{0}\right\|^{2}+ \\
k\left(\sum_{j=1}^{m} g_{j}(x)_{+}^{2}+\sum_{l=1}^{r}\left[h_{l}(x)\right]^{2}\right), \\
\quad \text { for all } k>0, \text { and } k \rightarrow \infty .
\end{gathered}
$$

Let $x^{k}$ be a solution of the problem

$$
\text { Min } \varphi_{k}(x) \text {, subject to }\left\|x-x^{0}\right\| \leq \delta \text {. }
$$

By the convergence property of penalty methods [15], we have

$$
\begin{gathered}
\mathfrak{F}\left(F\left(x^{k}\right)-F\left(x^{0}\right)\right)+\frac{1}{2}\left\|x^{k}-x^{0}\right\|^{2}+ \\
k\left\{\sum_{j=1}^{m} g_{j}(x)_{+}^{2}+\sum_{l=1}^{r}\left[h_{l}\left(x^{k}\right)\right]^{2}\right\} \\
\leq \mathfrak{F}\left(F\left(x^{0}\right)-F\left(x^{0}\right)\right)
\end{gathered}
$$

that is,

$$
\begin{aligned}
\mathcal{F}\left(F\left(x^{k}\right)-\right. & \left.F\left(x^{0}\right)\right)+\frac{1}{2}\left\|x^{k}-x^{0}\right\|^{2}+ \\
k & \left\{\sum_{j=1}^{m} g_{j}(x)_{+}^{2}+\sum_{l=1}^{r}\left[h_{l}\left(x^{k}\right)\right]^{2}\right\} \leq 0 .
\end{aligned}
$$


Suppose that $\mu_{j}^{k}=\left(k g_{j}\left(x^{k}\right)\right)_{+} \geq 0$ and $\tau_{l}^{k}=$ $k h_{l}\left(x^{k}\right)$, then we have

$$
\begin{aligned}
\mathcal{F}(F & \left.\left(x^{k}\right)-F\left(x^{0}\right)\right)+\frac{1}{2}\left\|x^{k}-x^{0}\right\|^{2} \\
& +\sum_{j=1}^{m}\left|\mu_{j}^{k} g_{j}\left(x^{k}\right)_{+}\right|+\sum_{l=1}^{r}\left|\tau_{l}^{k} h_{l}\left(x^{k}\right)\right| \leq 0 .
\end{aligned}
$$

By the convergence property of exact penalty methods [15], taking the $\lim _{k \rightarrow \infty} x^{k}=x^{0}, k \rightarrow \infty$ and by the continuity of $F$, we have

$$
\begin{aligned}
\lim _{x^{k} \rightarrow x^{0}}\left[\frac{1}{2}\left\|x^{k}-x^{0}\right\|^{2}+\right. & \sum_{j=1}^{m}\left|\mu_{j}^{k} g_{j}\left(x^{k}\right)_{+}\right| \\
& \left.+\sum_{l=1}^{r}\left|\tau_{l}^{k} h_{l}\left(x^{k}\right)\right|\right]=0 .
\end{aligned}
$$

In (6) we observe that $x^{k}$ exists because $\varphi_{k}(x)$ is continuous and $\bar{B}\left(x^{0}, \delta\right)$ is compact. Let $z$ be a limit point of $x^{k}$. We assume that $x^{k} \rightarrow z$. From the problem (5), we have

$$
\mathcal{F}\left(F\left(x^{k}\right)-F\left(x^{0}\right)\right) \leq \varphi_{k}\left(x^{k}\right),
$$

because of

$$
\begin{gathered}
\varphi_{k}\left(x^{k}\right)-\mathfrak{F}\left(F\left(x^{k}\right)-F\left(x^{0}\right)\right)= \\
\frac{1}{2}\left\|x^{k}-x^{0}\right\|^{2}+k\left\{\sum_{j=1}^{m} g_{j}(x)_{+}^{2}+\sum_{l=1}^{r}\left[h_{l}\left(x^{k}\right)\right]^{2}\right\} \geq 0 .
\end{gathered}
$$

Since $x^{*}$ is a feasible solution of the problem (4) and $x^{k}$ is the solution of problem (6), we have

$$
\varphi_{k}\left(x^{k}\right) \preceq_{L U} \varphi_{k}\left(x^{0}\right)=0 .
$$

We claim that $z$ is a feasible solution of the Problem (4). Since $\left\|x^{k}-x^{0}\right\| \leq \delta$, therefore $\left\|z-x^{0}\right\|<\delta$, suppose if possible

$$
\sum_{j=1}^{m}\left(g_{j}(z)_{+}\right)^{2}+\sum_{l=1}^{r} h_{l}^{2}(z)>0
$$

then, there exists $c>0$, such that

$$
\sum_{j=1}^{m}\left(g_{j}\left(x^{k}\right)_{+}\right)^{2}+\sum_{l=1}^{r} h_{l}^{2}\left(x^{k}\right)>c,
$$

for sufficiently large $\mathrm{k}$.
From continuity of $\mathfrak{F}$ and $x^{k} \rightarrow z$, we have

$$
\begin{aligned}
\varphi_{k}(x) & =\mathfrak{F}\left(F(x)-F\left(x^{0}\right)\right)+\frac{1}{2}\left\|x-x^{0}\right\|^{2} \\
+ & k\left\{\sum_{j=1}^{m} g_{j}\left(x^{k}\right)_{+}^{2}+\sum_{l=1}^{r}\left[h_{l}(x)\right]^{2}\right\} \\
& \succ_{L U} \mathfrak{F}\left(F(x)-F\left(x^{0}\right)\right)+k c .
\end{aligned}
$$

Taking the limit $k \rightarrow \infty$, we obtain $\varphi_{k}\left(x^{k}\right) \longrightarrow+\infty$, which contradicts (8). Consequently, $\sum_{j=1}^{m}\left(g_{j}(z)_{+}\right)^{2}+$ $\sum_{l=1}^{r} h_{l}^{2}(z)=0$, that is, $z \in P \cap \bar{B}\left(x^{0}, \delta\right)$, therefore from (7), we obtain

$$
\begin{array}{r}
\varphi_{k}\left(x^{k}\right)=\mathfrak{F}\left(F\left(x^{k}\right)-F\left(x^{0}\right)\right)+\frac{1}{2}\left\|x^{k}-x^{0}\right\|^{2} \\
\left.+k\left\{\sum_{j=1}^{m} g_{j}(z)_{+}\right)^{2}+\sum_{l=1}^{r}\left[h_{l}\left(x^{k}\right)\right]^{2}\right\} \leq 0, \\
\text { as } k \longrightarrow+\infty .
\end{array}
$$

Since $\left.k\left\{\sum_{j=1}^{m} g_{j}(z)_{+}\right)^{2}+\sum_{l=1}^{r}\left[h_{l}(x)\right]^{2}\right\} \geq 0$, therefore from (9), we have $\mathfrak{F}\left(F\left(x^{k}\right)-F\left(x^{0}\right)\right)+\frac{1}{2} \| x^{k}-$ $x^{0} \|^{2} \leq 0$. As $x^{0}$ is a unique solution of the problem (4), we conclude that $z=x^{0}$. Therefore, $x^{k} \longrightarrow x^{0}$ and $\left\|x^{k}-x^{0}\right\|<\delta$ for all $k$ sufficiently large. As $x^{k}$ is a solution of the nonsmooth problem (6) and it is an interior point of the feasible set, for sufficiently large $k$, from Proposition 2.3, it follows that $0 \in \partial^{c} \varphi_{\rho_{k}}\left(x^{k}\right)$. Then, we have

$$
\begin{aligned}
& 0 \in \operatorname{conv}\left(\bigcup_{i=1}^{p}\left\{F_{i}\left(x^{0}\right)\right\}\right)+\left(x^{k}-x^{0}\right) \\
& +\sum_{j=1}^{m} k g_{j}\left(x^{k}\right)_{+} \nabla g_{j}\left(x^{k}\right)+\sum_{l=1}^{r} k h_{l}\left(x^{k}\right) \nabla h_{l}\left(x^{k}\right) .
\end{aligned}
$$

Hence, there exists $\lambda_{i}^{k} \geq 0, i=1,2, \ldots, p$, such that $\sum_{i=1}^{p} \lambda_{i}^{k}=1$ and as $k g_{j}\left(x^{k}\right)_{+}=\mu_{j}^{k}, k h_{l}\left(x^{k}\right)=\tau_{l}^{k}$, then from (10), we get

$$
\begin{aligned}
& \sum_{i=1}^{p} \lambda_{i}^{L, k} F_{i}^{L}\left(x^{k}\right)+\sum_{i=1}^{p} \lambda_{i}^{L, k} F_{i}^{U}\left(x^{k}\right) \\
+ & \sum_{j=1}^{m} \mu_{j}^{k} \nabla g_{j}\left(x^{k}\right)+\sum_{l=1}^{r} \tau_{l}^{k} \nabla h_{l}\left(x^{k}\right)=x^{0}-x^{k} \rightarrow 0,
\end{aligned}
$$

as $x^{k} \longrightarrow x^{0}$ and $F_{i}\left(x^{k}\right) \rightarrow F_{i}\left(x^{0}\right)$. 
We established sufficient optimality conditions for the $V V I-I V O P$ problem.

\section{Theorem 3.2 Suppose}

$\left\langle F_{i}\left(x^{0}\right), x\right\rangle ;$ where each $F_{i}\left(x^{0}\right)$ $\left[F_{i}^{L}\left(x^{0}\right), F_{i}^{U}\left(x^{0}\right)\right], i=1, \cdots, p$ are $L U$-convex, $g_{j} ; j=1, \cdots, m$ are convex and $h_{i} ; i=1, \cdots, r$ are affine. If $x^{0} \in P$ satisfies the AKKT-VVI-IVOP conditions, then $x^{0}$ is a weak efficient solution of $V V I-I V O P$.

Proof: Let $x^{0}$ be not a weakly efficient solution then, there exists $\bar{x} \in P$ such that

$$
\left\langle F_{i}\left(x^{0}\right), \bar{x}\right\rangle \prec_{L U}\left\langle F_{i}\left(x^{0}\right), x^{0}\right\rangle, i=1,2, \ldots, p .
$$

Let $\left(x^{k}\right)$ and $\left(\lambda^{L, k}, \lambda^{U, k} \mu^{k}\right)$ be the sequences that satisfies the $A K K T-V V I-I V O P$ at $x^{0}$. Therefore, without loss of generality we may assume that $\lambda^{L, k} \rightarrow \lambda^{L, 0}, \lambda^{U, k} \rightarrow \lambda^{U, 0}$ with $\lambda^{L, 0} \geq 0, \lambda^{U, 0} \geq 0$ and $\sum_{i=1}^{p} \lambda_{i}^{L, 0}=1, \sum_{i=1}^{p} \lambda_{i}^{U, 0}=1$. As $\left\langle F_{i}\left(x^{0}\right), x\right\rangle$, are LU-convex, $g_{j}$ are convex and $h_{l}$ are affine, for all $k$ we get

$$
\begin{aligned}
&\left\langle F_{i}\left(x^{0}\right), x^{k}\right\rangle+\left\langle F_{i}\left(x^{k}\right), \bar{x}-x^{k}\right\rangle \preceq L U \\
&\left\langle F_{i}\left(x^{0}\right), \bar{x}\right\rangle, \forall i=1, \ldots, p,
\end{aligned}
$$

$$
g_{j}(\bar{x}) \geq g_{j}\left(x^{k}\right)+\left\langle\nabla g_{j}\left(x^{k}\right), \bar{x}-x^{k}\right\rangle, \forall j=1, \ldots, m,
$$

$$
h_{l}(\bar{x})=h_{l}\left(x^{k}\right)+\left\langle\nabla h_{l}\left(x^{k}\right), \bar{x}-x^{k}\right\rangle, \forall l=1, \ldots, m .
$$

Since $\bar{x}$ is feasible point, therefore we can write

$$
\begin{gathered}
\sum_{i=1}^{p} \lambda_{i}^{L, k}\left\langle F_{i}^{L}\left(x^{0}\right), \bar{x}\right\rangle+\sum_{i=1}^{p} \lambda_{i}^{U, k}\left\langle F_{i}^{U}\left(x^{0}\right), \bar{x}\right\rangle \\
+\sum_{j=1}^{m} \mu_{j}^{k} g_{j}(\bar{x})+\sum_{l=1}^{r} \tau_{l}^{k} h_{l}(\bar{x}) \\
\preceq_{L U} \sum_{i=1}^{p} \lambda_{i}^{L, k}\left\langle F_{i}^{L}\left(x^{0}\right), \bar{x}\right\rangle+\sum_{i=1}^{p} \lambda_{i}^{U, k}\left\langle F_{i}^{U}\left(x^{0}\right), \bar{x}\right\rangle .
\end{gathered}
$$

From (12), (13), (14) and (15), we get

$$
\begin{gathered}
\sum_{i=1}^{p} \lambda_{i}^{L, k}\left\langle F_{i}^{L}\left(x^{0}\right), x^{k}\right\rangle+\sum_{i=1}^{p} \lambda_{i}^{U, k}\left\langle F_{i}^{U}\left(x^{0}\right), x^{k}\right\rangle+ \\
\sum_{j=1}^{m} \mu_{j}^{k} g_{j}\left(x^{k}\right)+\sum_{l=1}^{r} \tau_{l}^{k} h_{l}\left(x^{k}\right) \\
+\left\langle\sum_{i=1}^{p} \lambda_{i}^{L, k} F_{i}^{L}\left(x^{0}\right)+\left\langle\sum_{i=1}^{p} \lambda_{i}^{U, k} F_{i}^{U}\left(x^{0}\right)\right.\right. \\
\left.+\sum_{j=1}^{m} \mu_{j}^{k} \nabla g_{j}\left(x^{k}\right)+\sum_{l=1}^{r} \tau_{l}^{k} \nabla h_{l}\left(x^{k}\right), \bar{x}-x^{k}\right\rangle \\
\preceq_{L U} \sum_{i=1}^{p} \lambda_{i}^{L, k}\left\langle F_{i}^{L}\left(x^{0}\right), \bar{x}\right\rangle+\sum_{i=1}^{p} \lambda_{i}^{U, k}\left\langle F_{i}^{U}\left(x^{0}\right), \bar{x}\right\rangle .
\end{gathered}
$$

Using $(A 1)-(A 3)$ in above inequality, we get

$$
\begin{aligned}
& \sum_{i=1}^{p} \lambda_{i}^{L, k}\left\langle F_{i}^{L}\left(x^{0}\right), x^{0}\right\rangle+\sum_{i=1}^{p} \lambda_{i}^{U, k}\left\langle F_{i}^{U}\left(x^{0}\right), x^{0}\right\rangle \\
& \preceq L U \sum_{i=1}^{p} \lambda_{i}^{L, k}\left\langle F_{i}^{L}\left(x^{0}\right)+\sum_{i=1}^{p} \lambda_{i}^{L, k}\left\langle F_{i}^{L}\left(x^{0}\right), \bar{x}\right\rangle,\right. \\
& \text { since } F_{i}\left(x^{k}\right) \rightarrow F_{i}\left(x^{0}\right) \text { as } x^{k} \rightarrow x^{0} .
\end{aligned}
$$

Which is a contradiction to (11). This completes the proof.

The $A K K T$ conditions are stronger than $K K T$ conditions in case of interval valued optimization. Here is an example of interval valued optimization problem which does not satisfy $K K T$ conditions but satisfy $A K K T$ conditions.

Example 3.1 Consider the following interval valued optimization problem:

$$
\min \phi(x)=[x+1, x+2]
$$

subject to $1+x^{2}=0$.

Example 3.2 Consider the following interval valued optimization problem:

$$
\begin{aligned}
& \text { Min } \phi\left(x_{1}, x_{2}\right)=\left(\phi_{1}\left(x_{1}, x_{2}\right), \phi_{2}\left(x_{1}, x_{2}\right)\right) \\
& \text { subject to } h\left(x_{1}, x_{2}\right)=x_{2}-x_{1}=0, \\
& \text { and } g\left(x_{1}, x_{2}\right)=x_{1}^{2}-x_{2} \leq 0, \\
& \text { where } \phi\left(x_{1}, x_{2}\right)=\left(\left[x_{1}-x_{2}^{2}+1, x_{1}-x_{2}^{2}+2\right],\right. \\
& \left.\left[x_{1}-x_{2}+1, x_{1}-x_{2}+2\right]\right) .
\end{aligned}
$$


The point $x^{0}=(1,1)$ is a weak efficient solution of the above problem. In order to find sequences satisfying the conditions (A1), (A2) and (A3), we solve the equation

$$
\begin{aligned}
\lambda_{1}^{L}\left[\begin{array}{c}
1 \\
-2 x_{2}
\end{array}\right]+ & \lambda_{2}^{L}\left[\begin{array}{c}
1 \\
-1
\end{array}\right]+\lambda_{1}^{U}\left[\begin{array}{c}
1 \\
-2 x_{2}
\end{array}\right] \\
& +\lambda_{2}^{U}\left[\begin{array}{c}
1 \\
-1
\end{array}\right] \\
& +\mu_{1} \nabla h\left(x_{1}, x_{2}\right)+\mu_{2} \nabla g\left(x_{1}, x_{2}\right)=(0,0) .
\end{aligned}
$$

Consider the sequence $x^{k}=\left(1+\frac{1}{k}, 1+\frac{1}{k}\right), k \in \mathbb{N}$, then

$\lambda_{1}^{L, k}=\left\{\frac{1}{24}+\frac{1}{k}\right\}, \lambda_{2}^{L, k}=\left\{\frac{19}{24}+\frac{1}{k}\right\}, \lambda_{1}^{U, k}=\left\{\frac{1}{12}+\right.$ $\left.\frac{1}{k}\right\}, \lambda_{2}^{U, k}=\left\{\frac{1}{12}+\frac{1}{k}\right\}$,

$\mu_{1}^{k}=\left\{\frac{5}{4}+\frac{1}{k}\right\}, \mu_{2}^{k}=\left\{\frac{1}{8}+\frac{1}{k}\right\}$.

Then we get

$$
\begin{gathered}
\lim _{k \rightarrow \infty} \lambda_{1}^{L, k} \nabla \phi_{1}^{L}\left(x_{1}^{k}, x_{2}^{k}\right)+\lambda_{2}^{L, k} \nabla \phi_{2}^{L}\left(x_{1}^{k}, x_{2}^{k}\right) \\
+\lambda_{1}^{U, k} \nabla \phi_{1}^{U}\left(x_{1}^{k}, x_{2}^{k}\right)+\lambda_{2}^{U, k} \nabla \phi_{2}^{U}\left(x_{1}^{k}, x_{2}^{k}\right) \\
+\mu_{1}^{k} \nabla h\left(x_{1}^{k}, x_{2}^{k}\right)+\mu_{2}^{k} \nabla g\left(x_{1}^{k}, x_{2}^{k}\right)=(0,0), \\
\sum_{i=1}^{p} \lambda_{i}^{L, k}+\sum_{i=1}^{p} \lambda_{i}^{U, k}=1, \\
\mu_{1}^{k} h\left(x^{k}\right)=\left(\frac{5}{4}+\frac{1}{k}\right) \times\left(1+\frac{1}{k}-1-\frac{1}{k}\right) \rightarrow 0, \\
\mu_{2}^{k} g\left(x^{k}\right)=\left(\frac{1}{8}+\frac{1}{k}\right) \times\left(\left(1+\frac{1}{k}\right)^{2}-1-\frac{1}{k}\right) \rightarrow 0 .
\end{gathered}
$$

Hence, AKKT - IVOP conditions are satisfied at $x^{0}=(1,1)$.

Example 3.3 Consider the following multiobjective optimization problem:

$$
\begin{gathered}
\text { Min } \phi\left(x_{1}, x_{2}\right)=\left(\phi_{1}\left(x_{1}, x_{2}\right), \phi_{2}\left(x_{1}, x_{2}\right)\right) \\
\text { subject to } h\left(x_{1}, x_{2}\right)=1-x_{1}-x_{2}=0, \\
\text { and } g\left(x_{1}, x_{2}\right)=2 x_{1}-x_{2}^{2}+1 \leq 0, \\
\text { where } \phi\left(x_{1}, x_{2}\right)=\left(\left[x_{2}-x_{1}^{2},-x_{2}-2 x_{1}^{2}\right],\right. \\
\left.\left[x_{2}-x_{1}, x_{1}-x_{2}+1\right]\right) .
\end{gathered}
$$

The point $x^{0}=(0,1)$ is a weak efficient solution of the above problem. In order to find sequences satisfying the conditions (A1), (A2) and (A3), we solve the equation

$$
\begin{aligned}
& \lambda_{1}^{L}\left[\begin{array}{c}
-2 x_{1} \\
1
\end{array}\right]+ \lambda_{2}^{L}\left[\begin{array}{c}
-1 \\
1
\end{array}\right]+\lambda_{1}^{U}\left[\begin{array}{c}
-4 x_{1} \\
-1
\end{array}\right] \\
&+\lambda_{2}^{U}\left[\begin{array}{c}
-1 \\
1
\end{array}\right] \\
&+\mu_{1}\left[\begin{array}{l}
-1 \\
-1
\end{array}\right]+\mu_{2}\left[\begin{array}{c}
2 \\
-2 x_{2}
\end{array}\right]=(0,0) .
\end{aligned}
$$

Consider the sequence $x^{k}=\left(\frac{1}{k}, 1+\frac{1}{k}\right), k \in \mathbb{N}$, then $\lambda_{1}^{L, k}=\left\{\frac{1}{48}+\frac{1}{k}\right\}, \lambda_{2}^{L, k}=\left\{\frac{43}{48}+\frac{1}{k}\right\}, \lambda_{1}^{U, k}=\left\{\frac{1}{24}+\right.$ $\left.\frac{1}{k}\right\}, \lambda_{2}^{U, k}=\left\{\frac{1}{24}+\frac{1}{k}\right\}$,

$\mu_{1}^{k}=\left\{\frac{85}{96}+\frac{1}{k}\right\}, \mu_{2}^{k}=\left\{\frac{1}{64}+\frac{1}{k}\right\}$.

Then we get

$$
\begin{gathered}
\lim _{k \rightarrow \infty} \lambda_{1}^{L, k} \nabla \phi_{1}^{L}\left(x_{1}^{k}, x_{2}^{k}\right)+\lambda_{2}^{L, k} \nabla \phi_{2}^{L}\left(x_{1}^{k}, x_{2}^{k}\right) \\
+\lambda_{1}^{U, k} \nabla \phi_{1}^{U}\left(x_{1}^{k}, x_{2}^{k}\right)+\lambda_{2}^{U, k} \nabla \phi_{2}^{U}\left(x_{1}^{k}, x_{2}^{k}\right) \\
+\mu_{1}^{k} \nabla h\left(x_{1}^{k}, x_{2}^{k}\right)+\mu_{2}^{k} \nabla g\left(x_{1}^{k}, x_{2}^{k}\right)=(0,0), \\
\sum_{i=1}^{p} \lambda_{i}^{L, k}+\sum_{i=1}^{p} \lambda_{i}^{U, k}=1, \\
\mu_{1}^{k} h\left(x^{k}\right)=\left(\frac{85}{96}+\frac{1}{k}\right) \times\left(1+\frac{1}{k}-1-\frac{1}{k}\right) \rightarrow 0, \\
\mu_{2}^{k} g\left(x^{k}\right)=\left(\frac{1}{64}+\frac{1}{k}\right) \times\left(\left(1+\frac{1}{k}\right)^{2}-1-\frac{1}{k}\right) \rightarrow 0 .
\end{gathered}
$$

Hence, AKKT - IVOP conditions are satisfied at $x^{0}=(0,1)$.

\section{Conclusions}

In this paper, we have studied the ApproximateKarush-Kuhn-Tucker $(A K K T)$ optimality conditions for interval valued optimization problem. We have provided an example which tells that $A K K T$ conditions are stronger than $K K T$ conditions. Further, we provided two more examples in the support of our theory. The further extension of this theory is possible in case of more generalized sequential optimality conditions namely, Complementary Approximate-KarushKuhn-Tucker $(C A K K T)$ optimality conditions [16].

Acknowledgements: The authors are indebted to anonymous referees for valuable comments and suggestions which led to the present improved version as it stands.

Funding: The second author is financially supported by CSIR-UGC JRF, New Delhi, India, through Reference no.: 1272/(CSIR-UGC NET DEC.2016). The 
third author is financially supported by Department of Science and Technology, SERB, New Delhi, India, through grant no.: MTR/2018/000121.

\section{References:}

[1] L. Fan, S. Liu and S. Gao, Generalized monotonicity and convexity of non-differentiable functions, J. Math. Anal. Appl. 279, 2003 pp. 279-289.

[2] H. C. Wu, The Karush-Kuhn-Tucker optimality conditions in an optimization problem with interval-valued objective function, European $J$. Oper. Res. 176(1), 2007 pp. 46-59.

[3] H. C. Wu, The Karush-Kuhn-Tucker optimality conditions in multiobjective programming problems with interval-valued objective functions, European J. Oper. Res. 196(1), 2009 pp. 49-60.

[4] H. C. Wu, On interval-valued nonlinear programming problems, J. Math. Anal. Appl. 338(1), 2008 pp. 299-316.

[5] R. E. Moore,Methods and Applications of Interval Analysis, SIAM Philadelphia 1994.

[6] A. Ben-Israel and P. D. Robers, A decomposition method for interval linear programming, Management Science 16(5), 1970 pp. 374-387.

[7] F. Giannessi, Theorems of alternative, quadratic programs and complementarity problems, Wiley, Chichester 1980.

[8] F. H. Clarke, Optimization and nonsmooth analysis, John Wiley \& Sons, Inc., New York 1983.

[9] G. Giorgi, B. Jiménez and V. Novo, Approximate Karush-Kuhn-Tucker condition in multiobjective optimization, J. Optim. Theory Appl. 171, 2016 pp. 70-89.

[10] G. Haeser and M. Schuverdt, On approximate KKT condition and its extension to continuous variational inequalities, J. Optim. Theory Appl. 149, 2011 pp. 528-539.

[11] G. Mastroeni, Some remarks on the role of generalized convexity in the theory of variational inequalities,Generalized convexity and optimization for economic and financial decisions Pitagora, Bologna 1999 pp. 271-281.

[12] H. W. Kuhn and A. W. Tucker, Nonlinear programming, University of California Press, Berkeley and Los Angeles 1951.

[13] J.-L. Lions and G. Stampacchia, Variational inequalities, Comm. Pure Appl. Math. 20, 1967 pp. 493-519.

[14] J. M. Martnez and B. F. Svaiter, A practical optimality condition without constraint qualifications for nonlinear programming, J. Optim. Theory Appl. 118, 2003 pp. 117-133.
[15] M. R. Hestenes, Optimization theory: the finite dimensional case, John Wiley \& Sons, New York 1975.

[16] R. Andreani, J. M. Martínez and B. F. Svaiter, A new sequential optimality condition for constrained optimization and algorithmic consequences, SIAM J. Optim. 20, 2010 pp. 35333554.

[17] R. Andreani, G. Haeser and J. M. Martínez, On sequential optimality conditions for smooth constrained optimization, Optimization 60, 2011 pp. 627-641.

[18] V. Laha and S. K. Mishra, On vector optimization problems and vector variational inequalities using convexificators, Optimization 66, 2017 pp. 1837-1850.

[19] J. Zhang, Q. Zheng, X. Ma and L. Li, Relationships between interval-valued vector optimization problems and vector variational inequalities, Fuzzy Optim. Decis. Making 15, 2016 pp. 33-55. 